\title{
Una Propuesta para la Elaboración de Guías de Práctica basadas en la Evidencia para Terapia Ocupacional
}

Aleida Fernández Moreno* Alix Solángel García Ruiz**

El interés por la calidad de la atención en salud se inició y se instauró desde comienzos de siglo; en los Estados Unidos, por la necesidad de crear estándares en los servicios. Posteriormente en otros países que han asumido el modélo norteamericano ha sido imperiosa la creación de sistemas que aseguren la calidad. Entre ellos, los protocolos, las guías de atención y las guías de práctica basadas en la evidencia ${ }^{1}$.

Por estos planteamientos y atendiendo al llamado de la Asóciación Colombiana de Terapia Ocupacional (ACTO), se pretende ofrecer en éste articulo lineamientos generales de cómo desarrollar una guía de práctica basada en la evidencia, al igual que el desarrollo de estas, tanto en el ámbito internacional como en el nacional.

Este documento presenta definiciones de guías de práctica basadas en la evidencia consideradas útiles para lograr servicios de calidad en Terapia Ocupacional que contribuyan al reconocimiento profesional en equipos interdisciplinarios. Se enfatiza en diferentes metodologías para el desarróllo de las guías, en particular la propues-

* Terapeuta Ocupacional. Mg. en Desarrollo Educativo y Social.

** Terapeuta Ocupacional. Mg. en Desarrollo Educativo y Social. Esp. en Seguridad Social.

1 Este aspecto puede ampliarse consultando el artículo Calidad en los servicios de Terapia Ocupacional y Guias de atención Basadas de la evidencia. Revista Ocupación Humana. 1998. 
ta de Mary Egan et al (1998) relacionadas con la sistematización de la práctica profesional.

\section{Guías de práctica basadas en la evidencia}

En la depuración conceptual de estrategias, los protocolos fueron remplazados por las guías de atención y esta a su vez superadas por las guías de práctica basadas en la evidencia, por ello a continuación se definen estas últimas.

En Colombia está ampliamente difundida la definición de la Asociación Colombiana de las Facultades de Medicina (Ascofame), que se refieren a las guías como "la generación de enunciados o principios que ayudan a los médicos y los pacientes a tomar decisiones acerca de los cuidados más apropiados en salud para las circunstancias clínicas específicas" 2 . Sin embargo, el énfasis clínico de esta definición frente a las particularidades de la Terapia Ocupacional obligan a indagar en la literatura propia sobre una conceptualización más coherente con el ejercicio de este profesional.

Para Sackett ét al (1996), las guías "son el uso concienzudo, explícito y juicioso de las mejores evidencias en la toma de decisiones acerca del cuidado de la salud de los individuos"3. En esta definición la práctica profesional marca la pauta sobre las mejores decisiones en cuanto al cuidado de un usuario, sin embargo, este planteamiento es de alguna manera superado y revisado por la Universidad de McMaster en Canadá el cual se apoyó en la "estrategia de aprendizaje clínico basado en problemas", siguiendo los lineamientos del Reino Unido ${ }^{4}$. Al incorporarse el término aprendizaje, se incluyó la idea del uso de literatura e investigación en la elaboración de guías.

2 ASOCIACIÓN COLOMBIANA DE FACULTADES DE MEDICINA - INSTITUTO DE SEGUROS SOCIALES. Diseño de guías de Práctica Clínica Basadas en la Evidencia. Santa Fe de Bogotá, Abril, 1997.

3 SACKETT ET AL (1996), citado por LLOYD - SMITHW. Evidence - based practice and Occupational Therapy. British Journal of Occupational Therapy. Vol. 60 No. 11 November, 1997.

4 BONTJE P. Trends in Occupational Therapy a worldwide perspective in. World Federation of Occupational Therapist. Bulletin. Vol. 37. May, 1998. 
Además de ser un requerimiento normativo (Ley 100/93 - Decreto reglamentario $1974 / 1996)^{5}$ el uso de guías de práctica basadas en la evidencia contribuye a desarrollar políticas y desarrollar servicios, acceder fácilmente al conocimiento de expertos, argumentar decisiones clínicas, respaldar decisiones administrativas y lograr reconocimiento profesional.

Al respecto, Lloyd - Smith (1997) presenta la evolución del cuidado de la salud, retomando a Gray:

\section{La evolución del cuidado de la salud basado en la evidencia}

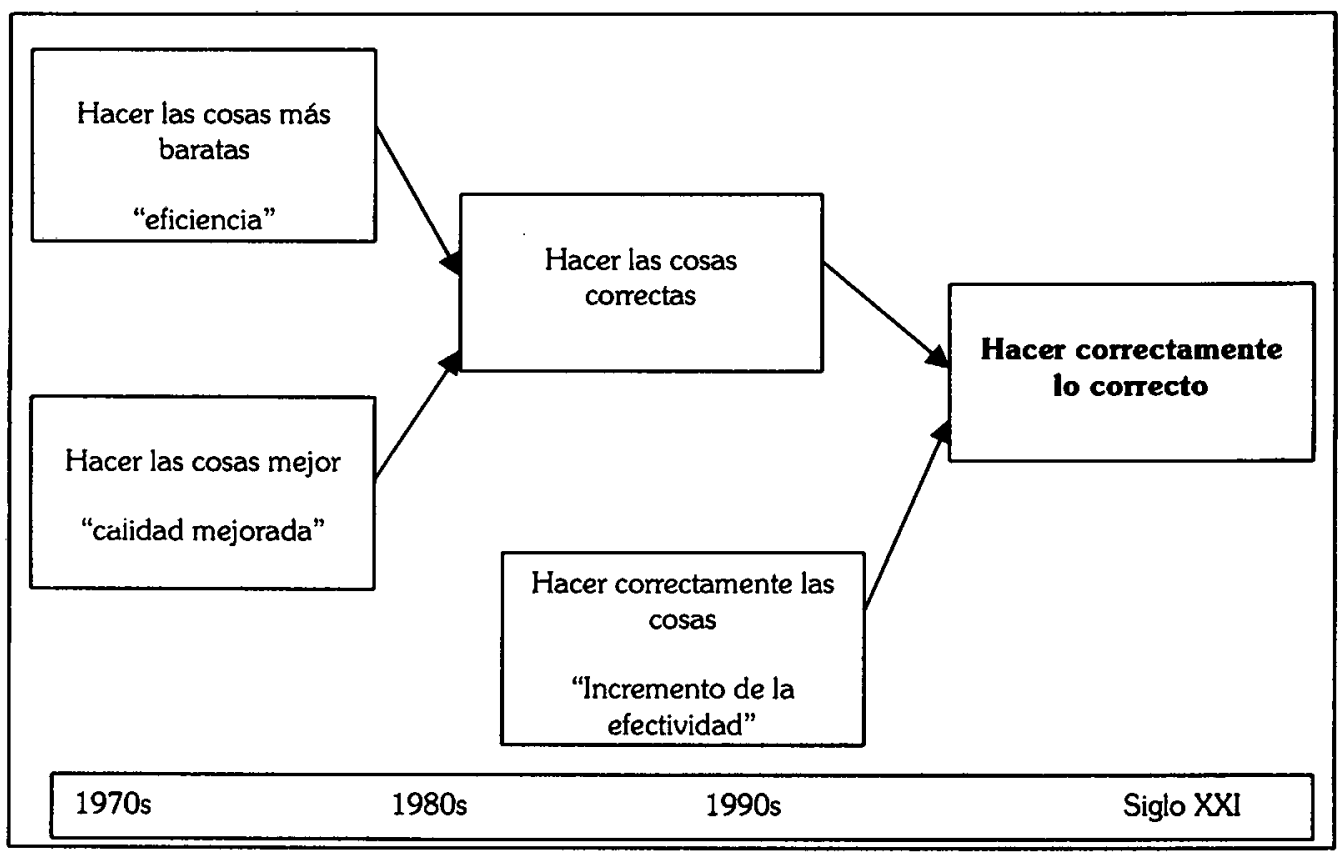

Fuente: GRAY Jam (1997) Evidence - based Healthcare. How to make Health Policy and Management Decisions. Edinburgh: Churchill Livingstone, p. 17.

5 El Decreto 1974 definió la calidad como "el conjunto de funciones, normas, requisitos y procedimientos indispensables para garantizar a los usuarios de los servicios el mayor beneficio a un costo razonable y con el menor riesgo posible". 
En síntesis, la elaboración de guías de práctica basadas en la evidencia es la posibilidad de interlocución entre los desarrollos logrados tanto en la investigación y la literatura profesional con la práctica para ser verificado, confrontado y replanteado en la prestación de los servicios. Así la guía de práctica basada en la evidencia es "una intervención basada sobre la revisión de la más creíble investigación encontrada y el consenso a partir de la opinión de expertos"6.

\section{¿Cómo hacer una guía de práctica basada en la evidencia?}

Existen diferentes propuestas metodológicas y aquí se hará mención a las principales.

La Asociación de Facultades de Medicina y el Instituto de Seguros Sociales ha realizado aproximadamente cuarenta guías de la práctica basada en la evidencia siguiendo esta metodología: (1) Decisiones preliminares que incluyen la estructura de la organización, la selección de tópicos, de grupo y definición de propósitos; (2) evaluación y resumen de la evidencia que incorpora selección de procedimientos e intervenciones, búsqueda de la evidencia en la literatura, evaluación de la calidad de la evidencia, síntesis de la evidencia, concepto de expertos y producción de una recomendación preliminar; (3) diseminación preliminar; (4) consideración de aspectos de la política aquí se toman en cuenta la limitación de aspectos humanos y económicos; (5) elaboración de la guía definitiva con recomendaciones para diseminación, evaluación, actualización e investigación; (6) adopción de la guía ${ }^{7}$.

Algunas guías en Terapia Ocupacional podrían seguir esta metodología si su contenido es clínico y se realiza en una institución hospitalaria. Sin embargo existen otras propuestas que deben considerarse como la de Alsop $(1997)^{8}$ quien retoma a Rosenberg y Donald (1995) y desarrolla los siguientes pasos: formular una pregunta clara, investigar en la literatura relevante, evaluar la evidencia para su validación

- EGAN, et al. The client - centred evidence- based practice of occupational therapy. Canadian Journal of Occupational Therapy practice of occupational therapy. Canadian Journal of Occupational Therapy. Vol. 65. No. 3. 1998.

7 ASOCIACIÓN COLOMBIANA DE FACULTADES DE MEDICINA - INSTITUTO DE SEGUROS SOCIALES. Diseño de guías de Práctica Clínica Basadas en la Evidencia. Santa Fe de Bogotá, Abril 1997.

8 ALSOP, Auldeen. Evidence - based practice and continuing professional development. British Journal of Occupational Therapy, Vol. 60. No. 11. 1997. 
e implementar én la práctica clínica. Vale decir que estos autores son los más citados en la literatura Británica de Terapià Ocupacional (Lloyd - Smith, 1997; Taylor, 1997; Alsop, 1997), ellos también son citados en la literatura Canadiense (Egan et al. 1998).

Las propuestas precedentes han tenido bastante reconocimiento y desarrollo en Colombia, sin embargo, se cree importante el aporte de Egan et al, (1998) terapeutas canadienses que desarrollan una metodología para la elaboración de guías de práctica basadas en la evidencia, diseño que será presentado a continuación.

\section{TIPOS DE EVIDENCIA REQUERIDOS EN EL MODELO DE TERAPIA OCUPACIONAL CANADIENSE}

\begin{tabular}{|c|c|c|}
\hline \multirow{2}{*}{$\begin{array}{l}\text { Etapas en el } \\
\text { proceso }\end{array}$} & \multicolumn{2}{|c|}{ Origen de la evidencia } \\
\hline & Basada en el cliente & Basada en la investigación \\
\hline $\begin{array}{l}\text { 1. Nombre } \\
\text { Validación- } \\
\text { Priorización de } \\
\text { problemas de } \\
\text { ejecución } \\
\text { ocupacional }\end{array}$ & $\begin{array}{l}\text { - Importancia de las ocupaciones } \\
\text { personales. } \\
\text { - Nivel de ejecución requerido. }\end{array}$ & $\begin{array}{l}\text { Inquietudes derivadas de la ejecución } \\
\text { ocupacional actual concerniente a los } \\
\text { componentes ocupacionales. } \\
\text {. }\end{array}$ \\
\hline $\begin{array}{l}\text { 2. Selección de } \\
\text { aproximaciones } \\
\text { teoricas }\end{array}$ & $\begin{array}{l}\text { Preferencia de aproximaciones } \\
\text { de resolución de problemas. }\end{array}$ & $\begin{array}{l}\text { - Marcos de referencia conceptuales } \\
\text { - Aplicabilidad y compatibilidad. }\end{array}$ \\
\hline $\begin{array}{l}\text { 3. Identificación } \\
\text { de componentes } \\
\text { de ejecución } \\
\text { ocupacional y } \\
\text { condiciones del } \\
\text { medio ambiente. }\end{array}$ & $\begin{array}{l}\text { - Reporte subjetivo de dificultades. } \\
\text { Interpretación medioambiental (ej. } \\
\text { grados de cambio posible). }\end{array}$ & $\begin{array}{l}\text { - Componentes de ejecución y factores } \\
\text { medioambientales cruciales para la } \\
\text { ejecución ocupacional. } \\
\text { Métodos asistenciales. } \\
\text { : }\end{array}$ \\
\hline $\begin{array}{l}\text { 4. Identificacion } \\
\text { de fuerzas y } \\
\text { recursos. }\end{array}$ & - Conocimiento de fuerzas y recursos. & $\begin{array}{l}\text { - Fuerzas y recursos que pueden ser. } \\
\text { apoyo de la ejecución ocupacional. } \\
\text { - Dinámica de proceso. }\end{array}$ \\
\hline $\begin{array}{l}\text { 5. Negociación de } \\
\text { objetivos y } \\
\text { desarrollo de plan } \\
\text { de acción. }\end{array}$ & $\begin{array}{l}\text { - Metas personales. } \\
\text { - Preferencia de estrategias de } \\
\text { resolución de problemas. }\end{array}$ & $\begin{array}{l}\text { Logros promedios de las metas de la } \\
\text { ejecución ocupacional dado en factores } \\
\text { ambientales y personales. }\end{array}$ \\
\hline $\begin{array}{l}\text { 6. Implementación } \\
\text { de planes a través } \\
\text { de la ocupación. }\end{array}$ & $\begin{array}{l}\text { - Ocupaciones preferidas } \\
\text { - Nivel personal de la intensidad del } \\
\text { apoyo requerido. }\end{array}$ & $\begin{array}{l}\text { Nivel promedio de intensidad de apoyo } \\
\text { requerido. }\end{array}$ \\
\hline $\begin{array}{l}\text { 7. Evaluación final } \\
\text { de la ejecución } \\
\text { ocupacional. }\end{array}$ & Nivel de logro de metas personales. & $\begin{array}{l}\text { - Métodos de evaluación del logro de } \\
\text { metas. }\end{array}$ \\
\hline
\end{tabular}

Fuente: EGAN et al. en CJOT (Revista Canadiense de Terapia Ocupacional) Junio de 1998. 
Priorizar los problemas del desempeño ocupacional es tener en cuenta el perfil epidemiológico y demográfico; la relevancia y competencia profesional; el ámbito tanto nacional como internacional; así como rescatar la importancia que la ocupación tiene para las personas, el nivel de ejecución que requiere -habilidades, capacidades frente a las demandas-y confrontar las inquietudes actuales de ejecución ocupacional (nivel de capacitación, desempleo, etc.). Hasta aquí se contextualizan los problemas del desempeño ocupacional.

La selección de aproximaciones teóricas requiere la revisión bibliográfica, la elección de un enfoque o marco de referencia, de instrumentos evaluativos, de insumos tecnológicos, así como el uso de terminología uniforme - propia de terapia ocupacional, todo ello para resolver los problemas ocupacionales detectados en la contextualización correlacionados con su aplicabilidad y compatibilidad en el contexto sociocultural donde se desarrolla la práctica. Es decir, se realiza una categorización donde se relacionan los problemas detectados con las posibilidades de solución desde la construcción teórica.

La identificación de componentes de ejecución ocupacional y las condiciones del medio ambiente necesitan la revisión de componentes de ejecución, la interpretación medioambiental y en general de factores medioambientales que inciden en la ocupación relacionados con los métodos asistenciales utilizados por el terapeuta. Esto es profundización, porque las aproximaciones teóricas previamente seleccionadas se confrontan con las particularidades ocupacionales colombianas, con los datos, con las condiciones culturales, con la singularidad de gustos e intereses de las personas en cuanto a su ocupación y los instrumentos que así lo requieran deberán ser adaptados a estas condiciones como soporte tecnológico que incide en la práctica profesional y en la calidad del servicio.

La identificación de fuerzas y recursos que pueden considerarse como apoyo o no de la ejecución ocupacional requieren la verificación de la dinámica del proceso; donde una vez se han discriminado los aspectos básicos de la experiencia se presentan ante el grupo profesional y se debate; así el equipo realizará posteriormente un plan de desarrollo que destaque los aspectos constitutivos de una guía basada en la evidencia. Esta sería una primera interpretación.

La negociación de objetivos y el desarrollo de un plan de acción tendrá en cuenta las metas personales, las estrategias en la solución de problemas, así como los logros ocupacionales según factores ambientales y personales. Estas deberán ser 
incoirporadas a la guía para destacar la complejidad y el dinamismo de la ocupación y evitar que estas se conviertan en una "receta". La propuesta preliminar de guía se debe, entonces, aplicar e implementar en los planes de tratamiento los cuáles requieren de un seguimiento donde nuevamente el uso de terminología uniforme, las estadísticas y el compartir los hallazgos con el equipo son importantes. Ellos pautan la confrontación inicial.

La implementación de planes a través de la ocupación teniendo en cuenta las ocupaciones preferidas, el soporte requerido con relación al nivel'de independencia personal. Esto es análisis y consenso siempre y cuando el equipo de trabajo halla sido consultado como una segunda interpretación sobre los avances y las determinaciones tomadas.

La evaluación final de la ejecución ocupacional incluye: los métodos de evaluación y el nivel de logro de métas personales y ocupacionales para que el grupo de expertos re - elabore la guía con esta información, disipe dudas con el apoyo de la revisión de literatura, se reflexione sobre los hallazgos de la guía preliminar y presente la guía de práctica basada en la evidencia ante su comunidad profesional. En esta etapa se habrá logrado la estructuración de la guía.

Las etapas presentadas por Egan et al (1998), fueron aquí tratadas, pero y teniendo en cuenta que para estos autores se deben determinar "cuáles decisiones son hechas en el curso de la terapia ocupacional y cuáles evidencias pueden impactar estas decisiones" creemos indispensable considerar tanto la divuilgación como la reestructuración de la guía, con ellas se buscan nuevos aportes de otros expertos y la retroalimentación necesaria para que la guía sea finalmente adoptada por los profesionales y perfeccionada por los mismos.

La contextualización; la categorización; profundización; interpretación; confrontación; análisis y consenso; estructuración; divulgación y réestructuración hacen parte de la propuesta de investigación en la práctica profesionà denominada sistematización. En particular retomá las etapas desarrolladas en la experiencia "filo de hambre" de Cendales y Mariño (1987) que ha sido revisada y adaptada por Fernández (1995). ${ }^{9}$

- La descripción de la sistematización como proceso investigativo ha sido desarrollada ampliamente por autores como Cadena (1987), Cendales y Mariño (1987), Ramírez Velázquez (1991), Mejía (1992), Aguilar (1994), Fernández (1995; 1999). 


\section{CONCLUSIONES}

- Las guías de práctica basadas en la evidencia son un soporte metodológico que contemplan aspectos teóricos, tecnológicos y éticos de la práctica, en este caso de la terapia ocupacional, lo que corresponde a "hacer correctamente lo correcto".

- La propuesta metodológica aquí planteada es un diálogo, una interlocución entre los avances teóricos e investigativos de la terapia ocupacional frente a la práctica profesional; lo cual no solo cumple la normatividad vigente sino desarrolla los servicios basados en principios de calidad.

- Por último se enfatiza en que el proceso de elaboración de guías de práctica basadas en la evidencia, es un compromiso colectivo y de consenso de la comunidad profesional de terapeutas ocupacionales. Este aspecto será desarrollado en un artículo posterior.

\section{BIBLIOGRAFÍA}

Alsop, A. Evidence - based practice and continuing professional development. British Journal of Occupational Therapy, Vol. 60 No. 11. 1997.

ASOCIACIÓN COLOMBIANA DE FACULTADES DE MEDICINA - INSTITUTO DE SEGUROS SOCIALES. Diseño de Guías de Práctica Clínica basadas en la Evidencia. Santa Fe de Bogotá, Abril 1997.

Bontje, P. Trends in Occupational Therapy a worldwide perspective in World Federation of Occupational Therapist. Bulletin. Vol. 37. May 1998.

Egan, M. et al. The client - centred evidence - based practice of occupational therapy. Canadian Journal of Occupational Therapy. Vol. 65 No. 3. 1998.

Lloyd - Smith W. Evidence - based practice and Occupational Therapy. British Journal of Occupational Therapy. Vol. 60 No. 11. November 1997. 


\section{BIBLIOGRAFÍA COMPLEMENTARIA}

Aguilar, Juan Francisco. Sistematización de Proyectos educativos Innovadores. Modulo 7. Maestría en Desarrollo Educativo y Social. CINDE. Santa Fe de Bogotá. 1994.

Cadena, Felix. La sistematización como proceso, como producto y como estructura de creación de saber. En: La sistematización en el trabajo de Educación Popular. Dimensión Educativa. Bogotá. 1987.

Cendales, Lola y Mariño, Germán. Anotaciones acerca de la sistematización. En: sistematización en èl trabajo de Educación Popular. Dimensión Educativa. Bogotá. 1987.

Mejía, Jorge. Sistematizar nuestras experiencias educativas. Materiales No. 10. CINEP. Santa Fe de Bogotá. 1992.

Ramírez Velásquez, Jorge Enrique. La sistematización espejo del maestro innovador. En: Cuadernos de Reflexión Educativa. CEPECS. Bogotá. 1991. 